\title{
The Blunt Abdominal Trauma Bedside Ultrasonography Comparison with Trauma Severity Scores and Computerized Tomography
}

\author{
Hizir Ufuk Akdemir, Fatih Caliskan, Celal Kati and Ahmet Baydin
}

\begin{abstract}
Objective: To investigate the accuracy of focussed assessment sonography for trauma (FAST) bedside ultrasonography application in patients with blunt abdominal trauma and patient management, clinical outcome, and trauma severity scores. Study Design: Cross-sectional descriptive study.

Place and Duration of Study: Department of Emergency Medicine, Ondokuz Mayis University, Faculty of Medicine, Samsun, Turkey, from April 2013 to June 2017.

Methodology: The presence of intraperitoneal free fluid (FF) was investigated by FAST application by emergency physicians. Abdominal computed tomography (CT), which was considered the gold standard, was made use of for evaluating intraperitoneal FF presence. Patients were evaluated for urgent surgical intervention, requirement of blood transfusion, and mortality status. The relationship between the FF presence / absence in FAST practice and revised trauma score (RTS), injury severity score (ISS) and trauma injury severity score (TRISS) was statistically analysed.

Results: All 28 FAST (+) patients also had intraperitoneal FF in gold standart CT. Although the gold standart CT detected intraperitoneal FF in $6(4.2 \%)$ of 140 FAST (-) patients, the compatibility between FAST and CT in the detection of intraperitoneal FF in patients with blunt abdominal trauma was statistically significant $(\mathrm{k}: 0.882, p<0.001)$. RTS and ISS scores were $6.24 \pm 1.74$ and $44.0 \pm 15.7$ in eleven $(3.5 \%)$ dead patients. The sensitivity, specificity, positive predictive value, and negative predictive value were found to be $82.3 \%$ (95\% Cl: $65.4-93.2), 100 \%$ (95\% Cl: $97.2-100), 100 \%$ and $95.7 \%(95 \%$ Cl: 91.5-97.8), respectively.

Conclusion: FAST had a high accuracy compared to gold standard CT in detecting FF. Low RTS and high ISS are associated with impaired hemodynamic parameters and detected FF [FAST (+)].
\end{abstract}

Key Words: Blunt, Abdominal, Trauma, Bedside, Ultrasonography, Emergency, Management.

\section{INTRODUCTION}

Trauma, defined as injury resulting from a mechanical force, is one of the leading causes of death in developed countries; and the most common cause of death in young adults between age 1 and 44 years. ${ }^{1}$ The majority of deaths following trauma occur at the scene or within the first four hours after arrival at a trauma centre. ${ }^{2}$ Most of these early post-traumatic deaths are related to hemorrhagic shock. Morbidity and mortality can be reduced by rapid identification of the traumatic patients requiring emergency and/or early surgical intervention. In the evaluation of post-traumatic hemorrhagic shock, focused assesment sonography for trauma (FAST) can be used to obtain important information on the etiology of the shock. About $15-20 \%$ of all trauma deaths are due to abdominal trauma. 3,4 Blunt abdominal trauma accounts for $80 \%$ of abdominal injuries, which can lead

Department of Emergency Medicine, Ondokuz Mayis University, Faculty of Medicine, Samsun, Turkey

Correspondence: Dr. Hizir Ufuk Akdemir, Department of Emergency Medicine, Ondokuz Mayis University, Faculty of Medicine, Samsun, Turkey

E-mail: hufukakdemir@hotmail.com

Received: October 12, 2018; Revised: January 14, 2019;

Accepted: January 15, 2019 to serious mortality and morbidity. 5 The use of invasive or non-invasive diagnostic methods is determined according to the hemodynamic status of patients with suspected intraabdominal bleeding following trauma. Ultrasonography (USG) and/or CT are used for diagnosis in hemodynamically stable patients with blunt abdominal trauma. In recent years, FAST has been frequently used in emergency departments to investigate the presence of intraabdominal bleeding, and it is increasingly important in the triage, diagnosis, and treatment modalities for the management of blunt trauma patients. FAST (Bedside USG) differs from CT as it does not require transfer of the patient to another unit from the emergency department (ED), and it can be used easily in hemodynamically unstable patients.

The aim of the present study was to investigate the relationship between the application of FAST performed by emergency physicians in patients with blunt trauma and the management, clinical outcome, prognosis, and trauma severity scores for such patients.

\section{METHODOLOGY}

This descriptive study was conducted at the Department of Emergency Medicine, Ondokuz Mayis University, Faculty of Medicine, Samsun, Turkey, from April 2013 to 
June 2017, with 315 patients admitted to the ED with blunt abdominal trauma. Inclusion criteria were patient admitted to the ED because of blunt trauma, of either gender, aged 18 years or older. Exclusion criteria were children $<18$ years with blunt abdominal trauma, patients who did not want to be involved in the study for any reason, patients who were admitted in the absence of trained personnel for FAST application, and diagnosed by CT only and without FAST.

The presence of intraperitoneal free fluid (FF) was investi-gated by performing FAST on patients enrolled in the study group, by emergency physicians (emergency medicine specialists and/or emergency medicine assistants). The criterion for certification to perform FAST within the scope of the study was that the practitioner had received theoretical and practical training. Siemens Acuson X300 PE Ultrasonography Device and the CH5-2 Curved Array Transducer (Convex Probe) were used for FAST application. The FAST exam evaluates three potential spaces within the peritoneal cavity for pathologic FF. The right upper quadrant (RUQ) visualizes the hepatorenal recess, also known as Morrison's pouch, the right paracolic gutter, the hepatodiaphragmatic area, and the caudal edge of the left liver lobe. The left upper quadrant (LUQ) visualizes the splenorenal recess, the subphrenic space, and the left paracolic gutter. Suprapubic images were used in order to evaluate FF in the rectovesical pouch in males and the rectouterine (Pouch of Douglas) and vesicouterine pouches in females. ${ }^{6}$ Detailed USG (USG Rad) for trauma was performed in the study patients by radiology department physicians (radiology specialists and/or radiology assistants).

Intravenous contrast-enhanced CT (gold standard CT) was used to assess the presence of intraperitoneal FF in patients with indications to obtain CT imaging by radiologists. ${ }^{3}$ The clinical status, trauma type and mechanism, and trauma scores of the patients evaluated by FAST application were recorded; as were the assessment of the need for urgent surgical intervention, the department that performed the surgical intervention, the blood and blood product transfusion requirement, information about hospitalization, the need for intensive care, and mortality. Patients with blunt abdominal trauma were divided into two groups according to FAST application: patients in whom FF was detected [FAST $(+)$ ] and patients without FF [FAST (-)].

Patients were also evaluated by the revised trauma score (RTS), the injury severity score (ISS), and the trauma and injury severity score (TRISS). The relationship between [FAST (+)] and [FAST (-)] patients and RTS, ISS, and TRISS was statistically analysed. After comparison of FAST and CT according to the presence of intraabdominal FF, the sensitivity, specificity, positive predictive value, and negative predictive value for FAST were calculated by diagnostic evaluation test.

Data were analysed with IBM SPSS V23 (Chicago, USA). Normal distribution of quantitative data was evaluated by Kolmogorov-Smirnov/Shapiro-Wilk tests. An independent samples t-test was used for comparison of normally distributed data. Mann Whitney U-test was used for comparison of non normally distributed data. The Kappa test was used when examining the compatibility of the methods. Quantitative data were presented as mean \pm standard deviation or median (min-max) and qualitative data as frequency (percent). The significance level was taken as $p<0.05$.

\section{RESULTS}

Two hundred and nineteen $(69.5 \%)$ of the patients were males and $96(30.5 \%)$ were females, and the mean age of the patients was $37.7 \pm 17.3$ years. Out of the 315 patients, $220(69.9 \%)$ had suffered traffic accidents, 64 $(20.3 \%)$ falls, and $31(9.8 \%)$ assaults. The average duration of hospitalization in the study group was 8.6 \pm 11.3 days. The patients' characteristics, hospitalization status, and surgery departments are given in Table I.

The distribution of FAST (+) and FAST (-) patients according to vital signs and trauma scoring systems (RTS, ISS, and TRISS) is given in Table II.

RTS score was $6.24 \pm 1.74$ in 11 patients who died due to trauma. There was a statistically significant difference between RTS and mortality $(p<0.001)$. RTS score was determined as $6.80 \pm 1.28$ in 28 patients who underwent surgery directly from ED. There was a statistically significant difference between RTS and undergoing emergency surgery $(p=0.009)$. Of 45 patients who were hospitalized to intensive care unit (ICU), RTS score was $6.71 \pm 1.35$. There was a statistically significant difference between RTS and ICU hospitalization $(p<0.001)$. RTS score was $7.78 \pm 0.27$ in 152 patients who were hospitalized in departments. There was a statistically significant difference between RTS and hospitalization ( $p=0.029)$. RTS score was determined as $7.82 \pm 0.15$ in 117 patients who were discharged from hospital. There was a statistically significant difference between RTS and discharge $(p=0.04)$.

ISS score was $44.0 \pm 15.7$ in 11 patients who died from trauma. There was a statistically significant difference between ISS and mortality $(p=0.026)$. Median of ISS score in 28 patients who underwent surgery directly from emergency department was 30 (11-71). There was no statistically significant difference between ISS and undergoing emergency surgery $(p=0.621)$. Median of ISS score in patients who were hospitalized in ICU was 36 (17-71). There was no statistically significant difference between ISS and ICU hospitalization $(p=0.600)$. ISS score was $18.06 \pm 9.54$ in 152 patients who were hospitalized in departments. There was a statistically significant difference between ISS and 
Table I: Patients' characteristics, hospitalization status, and surgery departments.

\begin{tabular}{|c|c|c|}
\hline & $\begin{array}{c}\text { Number } \\
\text { (n) }\end{array}$ & $\begin{array}{c}\text { Percent } \\
(\%)\end{array}$ \\
\hline \multicolumn{3}{|l|}{ Radiologist } \\
\hline Only USG performed & 147 & 46.6 \\
\hline Only CT performed & 162 & 51.4 \\
\hline CT performed after USG & 6 & 1.9 \\
\hline \multicolumn{3}{|l|}{ Gold standard CT - presence of FF } \\
\hline Absent & 134 & 42.5 \\
\hline Present & 34 & 10.8 \\
\hline \multicolumn{3}{|l|}{ Radiologist USG - presence of FF } \\
\hline Absent & 153 & 48.6 \\
\hline Present & 0 & 0 \\
\hline \multicolumn{3}{|l|}{ FAST operators } \\
\hline Emergency medicine assistant & 279 & 88.5 \\
\hline Emergency medicine specialist & 36 & 11.5 \\
\hline \multicolumn{3}{|l|}{ FAST - presence of FF } \\
\hline Absent & 287 & 91.1 \\
\hline Present & 28 & 8.9 \\
\hline \multicolumn{3}{|l|}{ Transfusion } \\
\hline Absent & 291 & 92.4 \\
\hline Present & 24 & 7.6 \\
\hline \multicolumn{3}{|l|}{ Hospitalization status } \\
\hline Not hospitalised & 118 & 37.5 \\
\hline Hospitalized & 197 & 62.5 \\
\hline \multicolumn{3}{|l|}{ Hospitalization departments } \\
\hline Emergency department & 32 & 10.1 \\
\hline \multicolumn{3}{|l|}{ General surgery } \\
\hline Department & 4 & 1.2 \\
\hline Intensive Care Unit & 14 & 4.4 \\
\hline Urology department & 2 & 0.6 \\
\hline \multicolumn{3}{|l|}{ Other } \\
\hline Department & 114 & 36.1 \\
\hline Intensive Care Unit & 31 & 9.8 \\
\hline Patients undergoing surgery & 95 & 30.1 \\
\hline Emergency department & 28 & 8.8 \\
\hline General surgery & 11 & 3.5 \\
\hline Other & 17 & 5.4 \\
\hline Surgery after hospitalization & 67 & 21.2 \\
\hline
\end{tabular}

${ }^{*}$ FF: Free fluid.

Table II: Distribution of [FAST (+)] and [FAST (-)] patients according to vital signs and trauma scoring systems.

\begin{tabular}{l|c|c}
\hline & $\begin{array}{c}\text { FAST }(+) \\
(\mathrm{n}=28)\end{array}$ & $\begin{array}{c}\text { FAST }(-) \\
(\mathrm{n}=287)\end{array}$ \\
\hline Systolic blood pressure $(\mathrm{mmHg})$ & $106 \pm 16.4$ & $114.6 \pm 10.6$ \\
Pulse (beat/minute) & $93.1 \pm 16.5$ & $79.3 \pm 10.4$ \\
Respiratory rate (breaths/minute) & $18.2 \pm 3.9$ & $14.4 \pm 3.4$ \\
RTS & $7.2 \pm 1.3$ & $7.7 \pm 0.6$ \\
ISS & $38.0 \pm 12.2$ & $15.4 \pm 10.8$ \\
TRISS & $0.7 \pm 0.3$ & $0.8 \pm 0.2$ \\
\hline
\end{tabular}

Table III: Distribution of patients according to the results of USG Rad, FAST, and Gold Standard CT.

\begin{tabular}{l|c|c}
\hline & $\begin{array}{c}\text { Gold Standard CT } \\
\text { FF negative }\end{array}$ & $\begin{array}{c}\text { Gold Standard CT } \\
\text { FF positive }\end{array}$ \\
\hline USG Rad - FF negative & 6 & 0 \\
USG Rad - FF positive & 0 & 1 \\
FAST (-) & 134 & 6 \\
FAST (+) & 0 & 28 \\
\hline
\end{tabular}

hospitalization $(p=0.009)$. ISS score was determined as $9.00 \pm 5.51$ in 117 patients who were discharged from emergency department. There was a statistically significant difference between ISS and those discharged from emergency department $(p=0.011)$.

One hundred and eighteen patients were not hospitalized and one of them died in emergency department. RTS and ISS score of this patient was 3.36 and 75 , respectively.

The distribution of patients according to the results of USG Rad, FAST, and the gold standard (CT) is given in Table III.

The compatibility between FAST and CT in the detection of intraperitoneal FF in patients with blunt abdominal trauma was statistically evaluated. According to the results obtained from the study group, FAST was strongly compatible with gold standard CT (Kappa: $0.882, p<0.001$ ) in respect of detecting FF presence. This study revealed that the sensitivity, specificity, positive predictive value, and negative predictive value were found to be $82.3 \%$ (95\% Cl: $65.4-93.2), 100 \%$ (95\% Cl: $97.2-100), 100 \%$ and $95.7 \%(95 \% \mathrm{Cl}: 91.5-$ 97.8), respectively. According to the results obtained from the present study group, true positive, false positive, true negative, and false negative values were $28,0,134$, and 0 , respectively.

Eleven $(3.5 \%)$ of the patients in the study group died (10 patients in ICU and 1 patient in ED). It was determined that the only exitus patient in the ED was a 20 -year male who was referred to the ED after being involved in a motor vehicle collision and who had massive hemopneumothorax, intraperitoneal free-fluid, and hepaticsplenic injury due to general body trauma; he died in the first hour of ED admission without any surgery. Four patients who underwent surgery after detection of intraperitoneal FF, died postoperatively at different times due to intensive care complications. Surgical treatment was performed to 3 of 5 patients who died from head trauma. The other one who died in ICU was an elderly woman who underwent chest tube due to pneumothorax related to chest trauma.

\section{DISCUSSION}

Abdominal trauma is responsible for $15-20 \%$ of all trauma deaths, and is the most frequent result of blunt injuries. 3,4 Abdominal trauma is related to increased morbidity and mortality. The main cause of mortality in patients with blunt abdominal trauma is hypovolemic shock. FAST is used to investigate the presence of intraperitoneal FF in the initial evaluation of trauma patients in emergency departments. ${ }^{7}$

Hsu et al. reported that FAST practice was performed by 17 different practitioners (seven emergency consultants, eight emergency doctors, and two general surgeons); 
and all of whom had the necessary theoretical and practical training and certification. ${ }^{7}$ In this study, the FAST practitioners were one emergency medicine specialist and six emergency medicine assistants. All practitioners had completed the necessary theoretical and practical training and had been certified.

The RTS is a physiological scoring system described by Champion et al. in 1988, adding respiratory rate and systolic blood pressure values to the calculated Glasgow Coma Scale score, and used to assess the current situation in trauma patients. ${ }^{8-10}$ Dur et al. reported that they found a strong association between RTS and mortality in their study involving 146 patients with multiple trauma, and mortality was seen starting from the RTS 7.1 threshold.9 Numerous studies with scoring systems have shown increased mortality and a need for intensive care in patients with low trauma scores.9,11-13 RTS scores were respectively $6.24 \pm 1.74$ and 6.71 \pm 1.35 in patients who developed mortality and admitted to the intensive care unit in this study group. The fact that the RTS values of FAST (+) patients were lower than those of FAST (-) patients in the present study was related to the deterioration of vital signs (hemodynamic instability). This is because the systolic blood pressure was lower in FAST (+) than in FAST (-) patients, and the other parameters of RTS, rate of pulse and respiration, were higher in FAST (+) than in FAST (-) patients.

The ISS is an anatomical scoring system that evaluates the injury according to the injured anatomical region. 14 In this scoring system, described by Baker, the patient's score is calculated by adding the squares of the highest three abbreviated injury scale (AIS) scores. A patient with an AIS score of 6 points in only one region is accepted as having the highest score of 75 . The ISS is inadequate to assess the severity of penetrating stab injuries. 15 Yagmur et al. compared the role of RTS and ISS in determining the likelihood of patients' survival in their study involving 72 multitrauma patients. ${ }^{16}$ This study found the mean value of ISS to be $9.99 \pm 8.58$ in patients who remained in departments, $26.87 \pm 21.84$ in patients discharged after hospitalization in ICU, and $48.78 \pm 22.98$ in patients who died in the ICU. ISS scores were respectively $44 \pm 15.7,36(17 ; 71)$, and $18.06 \pm 9.54$ in patients who developed mortality, admitted to the ICU and hospitalized in departments in this study group. In this study, the fact that ISS values were higher in FAST (+) patients than in FAST (-) patients was compatible with the literature data, and this was related to the deterioration in vital signs.

In a study involving 421 hemodynamically stable patients with blunt abdominal trauma, Dammer et al. compared the status of FAST (+) with ISS and poor clinical outcome (laparoscopy, laparotomy, angiographic embolization). ${ }^{17}$ They reported that a positive result of FAST at admission to the emergency service had a stronger association with poor clinical outcome than ISS and laboratory results. ${ }^{17}$ In approximately half of the patients who died in the present study group, the presence of FAST (+) was found to be associated with poor clinical outcomes.

Boyd et al. described the TRISS system by combining ISS, RTS, and an age factor (TRISS = RTS + ISS + A). ${ }^{18,19}$ TRISS is an anatomical and physiological scoring system and is better than other scoring systemes in predicting possible survival. The age of the patient is defined as "A"; if age is higher than $54, A=1$; if age is less than or equal to $54, A=0$ is assumed. When TRISS is calculated, not considering multiple injuries and existing chronic diseases in the same region reduces the effectiveness of this scoring system. Various studies have reported that TRISS provides more accurate information in determining the survival probability of patients. ${ }^{14,18,20}$ According to the results obtained from the present study, the TRISS values of FAST $(+)$ patients were lower than those of the FAST (-) patients.

Hsu et al., in their FAST study involving 410 patients with blunt abdominal trauma, reported that the true positive, false positive, true negative, and false negative values were 33, 0, 116, and 8, respectively. Differences between the studies may be explained by the fact that the results for all patients involved in the study by Hsu et al. were compared with a formal radiologist CT report; whereas, only 168 patients had CT evaluation in this study. ${ }^{7}$

Hsu et al. reported that the sensitivity, specificity, positive predictive value, and negative predictive value were $80 \%$ (95\% Cl: 73-88), 100\%, 100\% (95\% Cl: 85-94), and $94 \%$ (95\% Cl: 91-96), respectively. ${ }^{7}$ The differences between the studies were related to different study protocols (inclusion and exclusion criteria) and the different numbers of patients who received the gold standard (CT) used for the comparison.

The main limiting factors for this study were having different levels of experience of FAST practitioners, and patients who were diagnosed only by FAST but on whom CT was not performed (no indications for CT).

\section{CONCLUSION}

This study suggests that low RTS and high ISS are associated with impaired hemodynamic parameters and detected FF [FAST (+)] which are the indicators for hospitalization, a need for intensive care unit, and increased mortality. Early and appropriate FAST practice provides valuable and prognostic information. FAST prevents time delays and transportation out of the emergency department in the evaluation of hemodynamically unstable patients.

\section{REFERENCES}

1. http://www.nationaltraumainstitute.org/home/trauma_statistics.html. (National Trauma Institute Web site.) Accessed June 18, 2014. 
2. American College of Surgeons Committee on Trauma. Advanced Trauma Life Support (ATLS) Student Course Manual, 9th ed, American College of Surgeons, Chicago 2012.

3. French LK, Gordy S, Ma OJ. Abdominal Trauma. In: Tintinalli JE, Ma OJ, Yealy DM, Meckler GD, Cline DM, (Eds). Tintinalli's emergency medicine: A comprehensive study guide. ed. $8^{\text {th }}$, New York; Mc-Graw hill education; 2016: p.1761-64.

4. Demetriades D, Murray J, Charalambides K, Alo K, Velmahos G, Rhee $\mathrm{P}$, et al. Trauma fatalities: Time and location of hospital deaths. J Am Coll Surg 2004; 198:20-6.

5. Nishijima DK, Simel DL, Wisner DH, Holmes JF. Does this adult patient have a blunt intra-abdominal injury? JAMA 2012; 307:1517-27.

6. Chacko J, Brar G. Bedside ultrasonography applications in critical care: Part II. Indian J Crit Care Med 2014; 18:376-81.

7. Hsu JM, Joseph AP, Tarlinton LJ, Macken L, Blome S. The accuracy of focused assessment with sonography in trauma (FAST) in blunt trauma patients: Experience of an Australian major trauma service. Injury 2007; 38:71-5.

8. Yousefzadeh-Chabok S, Hosseinpour M, KouchakinejadEramsadati L, Ranjbar F, Malekpouri R, Razzaghi A, et al. Comparison revised trauma score, injury severity score and trauma and injury severity score for mortality prediction in elderly trauma patients. Ulus Travma Acil Cerrahi Derg 2016; 22:536-40.

9. Dur A, Cander B, Koçak S, Girisgin S, Gül M, Koyuncu F. Multiple trauma patients and trauma scoring systems inemergency-intensive care unit. JAEM 2009; 4:24-7.

10. Lichtveld RA, Spijkers AT, Hoogendoorn JM, Panhuizen IF, van der Werken $\mathrm{C}$. Triage revised trauma score change between first assessment and arrival at the hospital to predict mortality. Int J Emerg Med 2008; 1:21-6.

11. Orhon R, Eren SH, Karadayi S, Korkmaz I, Coskun A, Eren M, et al. Comparison of trauma scores for predicting mortality and morbidity on trauma patients. Ulus Travma Acil Cerrahi Derg 2014; 20:258-64.

12. Cevik Y, Dogan NÖ, Das M, Karakayali O, Delice O, Kavalci C. Evaluation of geriatric patients with trauma scores after motor vehicle trauma. Am J Emerg Med 2013; 31:1453-6.

13. Ahmad HN. Evaluation of revised trauma score in polytraumatized patients. J Coll Physicians Surg Pak 2004; 14: 286-9.

14. Galvagno SM Jr, Massey M, Bouzat P, Vesselinov R, Levy MJ, Millin MG, et al. Correlation between the revised trauma score and injury severity score: implications for prehospital trauma triage. Prehosp Emerg Care 2018; 1:1-8.

15. Cook A, Weddle J, Baker S, Hosmer D, Glance L, Friedman L, et al. A comparison of the injury severity score and the trauma mortality prediction model. J Trauma Acute Care Surg 2014; 76:47-52.

16. Yagmur Y, Güloglu C, Ugur M, Akkus Z, Çelik Y. Evaluation of patients with multiple injuries: Comparison of injury severity score and revised trauma score. Ulus Travma Acil Cerrahi Derg 1997; 3:73-77.

17. Dammers D, El Moumni M, Hoogland II, Veeger N, Ter Avest E. Should we perform a FAST exam in haemodynamically stable patients presenting after blunt abdominal injury: A retrospective cohort study. Scand J Trauma Resusc Emerg Med 2017; 25:1.

18. Jo S, Lee JB, Jin $\mathrm{YH}$, Jeong $\mathrm{T}$, Yoon J, Choi SJ, et al. Comparison of the trauma and injury severity score and modified early warning score with rapid lactate level (the ViEWS-L score) in blunt trauma patients. Eur $J$ Emerg Med 2014; 21:199-205.

19. Boyd CR, Tolson MA, Copes WS. Evaluating trauma care: The TRISS method: Trauma score and the injury severity score. J Trauma 1987; 27:370-8.

20. Raux M, Vivien B, Tourtier JP, Langeron O. Severity assessment in trauma patient. Ann Fr Anesth Reanim 2013; 32:472-6.

............ 